Año 11.

Frontera

Núm. 27
Revista de Investigación Académica sin

ISSN: 2007-8870

http://revistainvestigacionacademicasinfrontera.com

Recibido el 14 de abril de 2018. Dictaminado mediante arbitraje favorablemente 27 de junio de 2018

\title{
Ejercicio físico en mujeres embarazadas, efectos y consecuencias en parámetros antropométricos y cardiovasculares
}

\author{
Autores \\ Castro-Zamora Andrés Aquilino²; Borbón-Castro Norma Angélica ${ }^{2}$; Simental-Trinidad Jorge \\ Arturo $^{1}$; Félix-Ibarra Lucía Irene; Mendivil-Morales Miriam ${ }^{1}$; Oloño-Meza, Joel Alejandro ${ }^{3}$; \\ Durazo-Terán Luis Alberto ${ }^{2}$; Duarte-Robles Ana Patricia ${ }^{1}$
}

Autor de correspondencia Castro-Zamora, Andrés Aquilino ${ }^{1}$

Institución

Universidad Estatal de Sonora, Unidad Académica Navojoa ${ }^{1}$

Universidad Estatal de Sonora, Unidad Académica Hermosillo

Instituto Tecnológico de Sonora ${ }^{3}$ 
Año 11.

Frontera

Núm. 27
Revista de Investigación

Académica sin

ISSN: 2007-8870

\section{http://revistainvestigacionacademicasinfrontera.com}

\section{Resumen}

El ejercicio físico aunado a la nutrición adecuada ha demostrado contribuir en el rendimiento físico durante la práctica deportiva. El ejercicio físico planificado y dosificado a intensidades adecuadas durante el embarazo genera múltiples beneficios a la gestante y feto al mejorar el estilo de vida, minimiza los riesgos a la gestante, mejora aspectos psicológicos y sociológicos. Objetivo: analizar estudios científicos que describan efectos y consecuencias en parámetros antropométricos y cardiovasculares que se adquieren con la práctica del ejercicio físico en mujeres embarazadas. Materiales y métodos: estudio descriptivo y de revisión sistemática de artículos científicos, libros y capítulos de libros publicados en diversas bases de datos y centraban variables de investigación en mujeres que realizan ejercicio físico en las diversas etapas de gestación. El presente trabajo recopiló información científica publicada en los últimos diez años y que estaba relacionada con variables de ejercicio físico en embarazo, contraindicaciones y beneficios que ocasionan a la gestante y feto. Resultados: en la información analizada se recomienda ejercicio físico durante el embarazo a intensidades submáximas, principalmente con predominio aeróbico o a intensidades entre el 60 y $70 \%$ del consumo máximo de oxígeno. Entre las actividades más recomendadas se encuentran nadar, ejercicios de yoga, caminatas y bailes. Conclusiones: el ejercicio físico personalizado y supervisado por profesionales en el área deportiva repercute favorablemente aspectos cardiovasculares y antropométricos en la gestante y el feto, además contribuye a reducir riesgos de hipertensión, diabetes, obesidad y preeclampsia.

Palabras clave: gestante, ejercicio físico, antropometría y cardiovascular. 
Año 11.

Frontera

Núm. 27
Revista de Investigación

Académica sin

ISSN: 2007-8870

http://revistainvestigacionacademicasinfrontera.com

\section{Abstract}

Physical exercise in addition to an adequate nutrition has proven to contribute in performance during a sports practice. Scheduled and metered physical exercise in adequate intensities during pregnancy generates multiple benefits to a pregnant and the fetus, while improving the way of living, minimizes the pregnant risks, improves psychological and sociological aspects. Objective: Analyze scientific studies that describe the cardiovascular and anthropometric effects and consequences acquired on physical exercise practices with pregnant women. Methods and materials: Described study and systematic scientific articles revisions, books and chapters from published books in several databases and investigation variables targeted on women physical exercise practices during several pregnancy stages. This work gathered scientific information published within the last 10 years related to physical exercise on pregnancy, contraindications and benefits on a pregnant and the fetus. Results: The analyzed information recommends physical exercise during pregnancy on a sub-max level, mainly with aerobic exercises or between a $60 \%$ and $70 \%$ of maximum oxygen consumption. Among the most recommended activities are swimming, yoga exercises, walking and dancing. Conclusions: Personalized and professionally supervised physical exercise will positively improve cardiovascular and anthropometric within the pregnant and the fetus, it also contributes in hypertension, diabetes, obesity and preeclampsia reduction.

Key words: Pregnant, physical exercise, cardiovascular and anthropometric. 
Año 11.

Frontera

Núm. 27
Revista de Investigación

Académica sin

ISSN: 2007-8870

\section{http://revistainvestigacionacademicasinfrontera.com}

\section{Introducción}

El periodo de gestación es un estado biológico que tiene una duración aproximada de 280 días o 40 semanas, inicia con la fecundación del óvulo por el espermatozoide y termina con el parto de un neonato (Valera, 2009). Durante este proceso la gestante experimenta una serie de cambios fisiológicos naturales que inciden en procesos hormonales, físicos y alimentarios (Gaston y Prapavessis, 2013; Hernández, Quechol, Morales, y Pérez, 2005; Mendoza, Pérez, y Sánchez, 2010). Los cambios fisiológicos inician desde las primeras semanas de embarazo, incidiendo en el incremento de la FC entre 15 y 20 pulsaciones por minuto (ppm) y nuevamente entre 7-8 ppm durante el tercer trimestre debido al aumento del tamaño y desplazamiento a la izquierda que tiene el corazón para favorecer el desarrollo del feto (Broberg et al., 2015; González-Collado, Ruiz-Giménez, y Salinas-Salinas, 2013; Guelfi et al., 2015; Mourtakos et al., 2015; Onoyama et al., 2016; Sánchez García et al., 2016) ocasionando en las gestantes mejoras en el consumo de oxígeno y gasto metabólico, sin ningún riesgo en su salud (Aguilar et al., 2014).

El ejercicio físico (EF) es considerado una parte de la actividad física (AF) y refiere a los movimientos planificados y repetidos que realiza un individuo con la finalidad de mejorar el estado de salud (Weinberg, 2010). La práctica regular de EF genera múltiples beneficios en el organismo, principalmente cuando se realiza por debajo del $70 \%$ del consumo máximo de oxígeno $\left(\mathrm{VO}_{2}\right.$ máx) al relacionarse con la disminución de grasa corporal y aumento de la resistencia aeróbica influyendo en la mejora de la calidad de vida (Constantinou, Oddie, y Watson, 2015; Hernández García, y Rivas Estany, 2016; Pescatello, Arena, Riebe, y Thompson, 2013).

En caso de mujeres que se encuentren en periodo de embarazo "gestación", se ha demostrado que el EF presenta beneficios en variables cardiovasculares y antropométricas, favoreciendo directamente a la gestante, embrión y feto (Guelfi et al., 2015; Mata et al., 2010; Muñoz et al., 
Año 11.

Frontera

Núm. 27
Revista de Investigación

Académica sin

ISSN: 2007-8870

\section{http://revistainvestigacionacademicasinfrontera.com}

2016; Onoyama et al., 2016; Sánchez et al., 2016). En las gestantes incluyen aumento en el $\mathrm{VO}_{2}$ máx, disminución de la frecuencia cardiaca (FC), mantenimiento del peso corporal (PC), principalmente en masa grasa (MG), mayor irrigación sanguínea, disminución de calambres e inflamación en piernas y mejoras en la postura (Baños, 2016; Toirac et al., 2010). En el feto se disminuye la probabilidad de padecer hiperglucemia después de su nacimiento, mejora su crecimiento y en caso de realizarse EF vigoroso se produce un aumento en las células endoteliales permitiéndole mejor condición cardiovascular (Smith, Foster, y Campbell, 2011; Deierlein, Siega-Riz, Everson, 2012).

La práctica de EF se recomienda antes del periodo de gestación por las adaptaciones orgánicas que se adquieren (Serrano y García, 2009; Rodriguez-Blanque et al., 2017; Oliveria Melo, 2012). Para esto, los expertos en el área médica y deportiva valoran la aptitud física antes de iniciar un programa de acondicionamiento físico con la finalidad de dosificar las cargas de entrenamiento, favorecer la salud física de la gestante y feto (Moyer, Livingston, Fang, May, y 2015). El Colegio Estadounidense de Obstetras y Ginecólogos "American Congress of Obstetricians and Gynecologists (ACOG)" recomienda que las gestantes realicen EF por debajo del 70\% de la FCmáx “intensidad moderada”, tres veces por semana con una duración entre 3040 minutos por sesión (Sánchez et al., 2016). Aunque la intensidad de entrenamiento puede incrementar dos o tres veces por semana según la adaptación cardiovascular que presente la gestante (Fagevik Olsen, Glantz y Petrov Fieril, 2015).

Las recomendaciones realizadas para la práctica de EF en periodo de gestación están centradas en las modificaciones biológicas que se generan en la gestante, tales como los cambios en las dimensiones pulmonares, desplazamiento del diafragma hacia el tórax por la expansión del útero, desplazamiento y aumento del tamaño del corazón, incremento de las curvaturas de la columna vertebral (Broberg et al., 2016; Ewton y May, 2017; Feldens et al., 2010; Martínez, 2016; Richardsen et al., 2016; Valdés y Carvajal, 2016). 
Año 11.

Frontera

Núm. 27
Revista de Investigación

Académica sin

ISSN: 2007-8870

\section{http://revistainvestigacionacademicasinfrontera.com}

En un estudio realizado por Guelfi, et al., (2016) sometieron a mujeres embarazadas a EF interválico, realizado en bicicleta estacionaria a intensidades sub-máximas presentándose tolerancia y disfrute por parte de las gestantes cuando se realiza con estas características, teniendo como consecuencia mejoras en el control de la glucosa postprandial. A pesar de la información surgida en los últimos 10 años (Guelfi, et al., 2016; Sánchez et al., 2016; Oliveria Melo, 2012; Rodriguez-Blanque et al., 2017) sobre los beneficios de realizar EF a diversas intensidades y modalidades, con regularidad las mujeres en periodo de gestación disminuyen esta práctica, por los paradigmas que aún existen sobre efectos negativos que pudiera ocasionar al feto (Ong et al., 2016), tales como asociar el ingreso de lactantes a unidades de cuidados intensivos neonatales por parto pretérmino, bajo peso al nacer o de restricción del crecimiento intrauterino (Aguilar et al., 2014). Por lo tanto, el objetivo del presente estudio fue identificar los beneficios que presenta el EF en parámetros antropométricos y cardiovasculares durante el periodo de gestación.

\section{Materiales y métodos}

Tipo de estudio

Se realizó un estudio descriptivo y de revisión sistemática de artículos científicos, libros y capítulos de libros publicados en diversas bases de datos, los cuales centraban sus variables de investigación en mujeres que realizan EF en los diversos trimestres de gestación.

Selección de los artículos y estrategia de búsqueda

Se identificó a través de la búsqueda automatizada en bases de datos Scopus, PubMed, Google Scholar, Aol search y Gibiru. La revisión de la información se efectuó entre los meses de septiembre de 2017 a febrero de 2018. Como estrategia de búsqueda se limitó la recopilación de artículos científicos publicados con fecha 2008-2018. 
Año 11.

Frontera

Núm. 27
Revista de Investigación

Académica sin

ISSN: 2007-8870

\section{http://revistainvestigacionacademicasinfrontera.com}

Los descriptores o palabras clave de búsqueda utilizadas fueron: mujeres embarazadas, actividad física, ejercicio físico, deporte y embarazo. Estos mismos fueron utilizados en idioma ingles: pregnant, pregnancy, physical activity, exercise and sport and pregnancy. Para conocer la terminología correcta se consultó el diccionario de la real academia española y posteriormente las palabras fueron traducidas al idioma inglés.

La información analizada y sintetizada se colocó en casillas que describían nombre de artículo, año de publicación, objetivo de estudio, palabras claves, teoría principal, metodología, resultados más importantes, principales discusiones, conclusiones y autores más citados.

El proceso de búsqueda permitió seleccionar 115 artículos de los cuales 20 se utilizaron para resultados, los cuales cumplieron con los criterios establecidos para la redacción del presente estudio. La información más relevante se muestra en la sección de resultados (Tablas, 1 y 2). 


\section{Académica sin Frontera}

ISSN: 2007-8870

\section{http://revistainvestigacionacademicasinfrontera.com}

\section{Revista de Investigación}

\section{Resultados}

Tabla 1. Principales efectos cardiovasculares que ocasiona el ejercicio físico en mujeres gestantes.

\begin{tabular}{|c|c|c|c|c|c|}
\hline Objetivo & Muestra & $\begin{array}{c}\text { Principales } \\
\text { resultados }\end{array}$ & $\begin{array}{l}\text { Intensidad de } \\
\text { entrenamiento }\end{array}$ & $\begin{array}{l}\text { Principales } \\
\text { conclusiones }\end{array}$ & $\begin{array}{l}\text { Autor/es y } \\
\text { año }\end{array}$ \\
\hline $\begin{array}{l}\text { Analizar la influencia de un programa } \\
\text { de AF moderada para la gestante en el } \\
\text { medio acuático y su relación con la } \\
\text { ventilación pulmonar. }\end{array}$ & $\begin{array}{l}140 \text { gestantes } \\
\text { sanas, entre } 21 \text { y } \\
43 \text { años. }\end{array}$ & $\begin{array}{l}\text { El EF mejora la CF de } \\
\text { las gestantes y facilitó } \\
\text { la respiración. }\end{array}$ & Moderada & $\begin{array}{l}\text { El EF en medio acuático no } \\
\text { compromete la oxigenación } \\
\text { pulmonar materna ni la entrega } \\
\text { directa de oxígeno al útero. }\end{array}$ & $\begin{array}{l}\text { Rodríguez- } \\
\text { Blanque et al., } \\
2017 .\end{array}$ \\
\hline $\begin{array}{l}\text { Evaluar en gestantes saludables el } \\
\text { efecto del EF aeróbico sobre el } \mathrm{VO}_{2} \text {. }\end{array}$ & $\begin{array}{l}64 \text { gestantes } \\
\text { saludables, entre } \\
20 \text { y } 28 \text { años. }\end{array}$ & $\begin{array}{l}\text { Las participantes } \\
\text { mejoraron la } \mathrm{CF} \\
\text { respiratoria. }\end{array}$ & Moderada & $\begin{array}{l}\text { El EF aeróbico en embarazadas } \\
\text { puede ser una alternativa para } \\
\text { prevenir patologías relacionadas } \\
\text { con el sedentarismo. }\end{array}$ & Vega et al., 2017. \\
\hline $\begin{array}{l}\text { Evaluar los niveles de AF en } \\
\text { gestantes y examinar las } \\
\text { características asociadas con la } \\
\text { práctica del EF y las actividades } \\
\text { de la vida diaria. }\end{array}$ & $\begin{array}{l}1.279 \text { gestantes } \\
\text { dentro de las } 72 \\
\text { horas postparto. }\end{array}$ & $\begin{array}{l}\text { El EF disminuye en el } \\
\text { embarazo, la caminata } \\
\text { fue el EF más común } \\
\text { seguido de EF acuático. }\end{array}$ & baja & $\begin{array}{l}\text { Promover el EF en el embarazo } \\
\text { sigue siendo un factor importante } \\
\text { que necesita ser implementado } \\
\text { como políticas en salud pública. }\end{array}$ & $\begin{array}{l}\text { Nascimento et al., } \\
2015 .\end{array}$ \\
\hline $\begin{array}{l}\text { Establecer a que intensidad de EF } \\
\text { (ciclismo estacionario) exige mayor } \\
\mathrm{VO}_{2} \text {, y gasto de energía. }\end{array}$ & $\begin{array}{l}12 \text { gestantes } \\
\text { sanas con } 30 \\
\text { semanas y edades } \\
\text { de entre } 35 \text { y } 36 \\
\text { años. }\end{array}$ & $\begin{array}{l}\text { La FC, el VO2 y el gasto } \\
\text { de energía fueron más } \\
\text { demandantes durante los } \\
\text { primeros } 15 \mathrm{~min} \text { de haber } \\
\text { empezado el EF en } \\
\text { bicicleta. }\end{array}$ & Moderada & $\begin{array}{l}\text { Es necesaria más información para } \\
\text { determinar si estos valores } \\
\text { influyen de manera positiva en la } \\
\text { embarazada que realiza EF. }\end{array}$ & Ong et al., 2016. \\
\hline
\end{tabular}

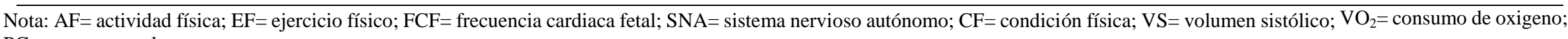
$\mathrm{PC}=$ peso corporal. 


\section{Académica sin Frontera}

ISSN: 2007-8870

\section{http://revistainvestigacionacademicasinfrontera.com}

\section{Revista de Investigación}

Principales efectos cardiovasculares que ocasiona el ejercicio físico en mujeres gestantes (continuación Tabla 1).

\begin{tabular}{|c|c|c|c|c|c|}
\hline Objetivo & Muestra & $\begin{array}{c}\text { Principales } \\
\text { resultados }\end{array}$ & $\begin{array}{l}\text { Intensidad de } \\
\text { entrenamiento }\end{array}$ & $\begin{array}{c}\text { Principales } \\
\text { conclusiones }\end{array}$ & $\begin{array}{l}\text { Autor/es y } \\
\text { año }\end{array}$ \\
\hline $\begin{array}{l}\text { Describir los principales resultados } \\
\text { maternos y perinatales asociados a la } \\
\text { preclamsia grave y la AF. }\end{array}$ & $\begin{array}{l}377 \text { gestantes de } \\
18 \text { años y mayores } \\
\text { de } 35 \text { años. }\end{array}$ & $\begin{array}{l}\text { El EF evita posible } \\
\text { aparición de preeclamsia } \\
\text { y aumento del PC en la } \\
\text { gestante. }\end{array}$ & moderada & $\begin{array}{l}\text { La preeclamsia puede ocasionar } \\
\text { muerte al feto, programas de EF en } \\
\text { el embarazo puede contrarrestar } \\
\text { estos efectos. }\end{array}$ & $\begin{array}{l}\text { Suárez González } \\
\text { et al., } 2009 .\end{array}$ \\
\hline $\begin{array}{l}\text { Evaluar el efecto y la seguridad del EF } \\
\text { de resistencia moderada a vigorosa } \\
\text { durante el embarazo. }\end{array}$ & $\begin{array}{l}\text { Mujeres entre } 14 \text { y } \\
15 \text { semanas de } \\
\text { embarazo, con AF } \\
\text { rigurosa. }\end{array}$ & $\begin{array}{l}\text { Disminución de estrés, } \\
\text { dolor de espalda y } \\
\text { estabilidad en la PA. }\end{array}$ & Moderada- Alta & $\begin{array}{l}\text { El EF moderado e intenso } \\
\text { supervisado no pone en riesgo el } \\
\text { crecimiento ni desarrollo fetal. }\end{array}$ & $\begin{array}{l}\text { Petrov et al., } \\
2015 .\end{array}$ \\
\hline $\begin{array}{l}\text { Determinar los efectos de diferentes } \\
\text { modos de entrenamiento (aeróbico, } \\
\text { circuito y resistencia) durante todo } \\
\text { el embarazo, así como adaptaciones } \\
\text { fetales que se presentan. }\end{array}$ & $\begin{array}{l}\text { Mujeres entre } 16 \text { y } \\
36 \text { semanas de } \\
\text { embarazo, con } \\
\text { edades de } 20 \text { a } 29 \\
\text { años. }\end{array}$ & $\begin{array}{l}\text { El feto no está en } \\
\text { riesgo de hipoxia o } \\
\text { bradicardia durante el } \\
\text { EF aeróbico, en EF de } \\
\text { circuito y resistencia el } \\
\text { feto modula la FC y se } \\
\text { adapta de manera } \\
\text { similar al corazón } \\
\text { adulto cuando se } \\
\text { expone al EF. }\end{array}$ & Moderada-Alta & $\begin{array}{l}\text { Es escasa la información sobre las } \\
\text { adaptaciones y los beneficios del } \\
\text { EF materno a diversas intensidades } \\
\text { de trabajo, principalmente en el } \\
\text { desarrollo y función } \\
\text { cardiovascular. }\end{array}$ & $\begin{array}{l}\text { Moyer et al., } \\
2015 .\end{array}$ \\
\hline $\begin{array}{l}\text { Examinar si la participación en un } \\
\text { programa de EF durante el embarazo } \\
\text { se asocia con mejoras en el bienestar } \\
\text { psicológico y físico entre mujeres } \\
\text { previamente inactivas. }\end{array}$ & $\begin{array}{l}66 \text { gestantes con } \\
\text { edades de } 30 \text { a } 34 \\
\text { años. }\end{array}$ & $\begin{array}{l}p<0.05 \text { en el humor, PA, } \\
\text { TA, fatiga y } \\
\text { rendimiento físico. }\end{array}$ & Moderada & $\begin{array}{l}\text { Es importante continuar } \\
\text { promoviendo el EF durante el } \\
\text { embarazo. }\end{array}$ & $\begin{array}{l}\text { Gaston y } \\
\text { Prapavessis, } \\
2013 \text {. }\end{array}$ \\
\hline
\end{tabular}

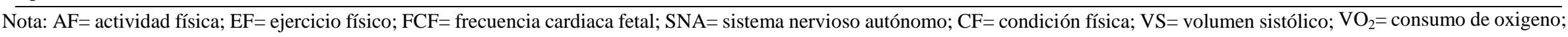
$\mathrm{PC}=$ peso corporal. 
Año 11.

\section{Académica sin Frontera}

Revista de Investigación

Núm. 27

ISSN: 2007-8870

http://revistainvestigacionacademicasinfrontera.com

Principales efectos cardiovasculares que ocasiona el ejercicio físico en mujeres gestantes (continuación Tabla 1).

\begin{tabular}{|c|c|c|c|c|c|}
\hline Objetivo & Muestra & $\begin{array}{c}\text { Principales } \\
\text { resultados }\end{array}$ & $\begin{array}{l}\text { Intensidad de } \\
\text { entrenamiento }\end{array}$ & $\begin{array}{c}\text { Principales } \\
\text { conclusiones }\end{array}$ & $\begin{array}{c}\text { Autor/es y } \\
\text { año }\end{array}$ \\
\hline $\begin{array}{l}\text { Estimar el efecto del EF supervisado } \\
\text { en la AF de la madre, el flujo } \\
\text { sanguíneo feto- placentario y el } \\
\text { crecimiento fetal. }\end{array}$ & $\begin{array}{l}3 \text { grupos de } \\
\text { embarazadas que } \\
\text { realizan EF. } \\
\text { A) } 13 \text { semanas de } \\
\text { gestación. } \\
\text { B) } 20 \text { semanas. } \\
\text { C) Sin } \\
\text { supervisión. }\end{array}$ & $\begin{array}{l}\text { La CF mejoró para todos } \\
\text { los grupos después de } \\
\text { las } 28 \text { semanas, con } \mathrm{VO}_{2} \\
\text { promedio. No se } \\
\text { encontró asociación de } \\
\text { la práctica de } \mathrm{EF}, \\
\text { con las variables } \\
\text { investigadas. }\end{array}$ & Moderada & $\begin{array}{l}\text { EF intensidad moderada mejoró } \\
\text { satisfactoriamente la condición } \\
\text { física de las gestantes que } \\
\text { anteriormente habían sido } \\
\text { sedentarias sin afectar el flujo } \\
\text { sanguíneo feto-placentario ni el } \\
\text { crecimiento fetal. }\end{array}$ & $\begin{array}{l}\text { Oliveria et al., } \\
2012 \text {. }\end{array}$ \\
\hline $\begin{array}{l}\text { Valorar la influencia de un programa } \\
\text { de EF durante la gestación en la } \\
\text { adaptación de la } \\
\text { FCF. }\end{array}$ & $\begin{array}{l}45 \text { gestantes en } 3^{\mathrm{er}} \\
\text { trimestre se midió } \\
\text { la FCF post-EF. }\end{array}$ & $\begin{array}{l}\text { El EF mejoró la } \\
\text { adaptación de FCF } \\
\text { post-EF y recupera } \\
\text { del parto más rápido. }\end{array}$ & Moderada & $\begin{array}{l}\text { Los programas de EF son } \\
\text { beneficiosos en mujeres } \\
\text { embarazadas y feto. }\end{array}$ & $\begin{array}{l}\text { Roldán, Perales, } \\
\text { Mateos y Barakat, } \\
2015 .\end{array}$ \\
\hline $\begin{array}{l}\text { Conocer la influencia de un programa } \\
\text { de EF supervisado en la FCF en } \\
\text { reposo y después del } \\
\text { EF materno en relación con } \\
\text { gestantes sedentarias. }\end{array}$ & $\begin{array}{l}81 \text { gestantes } \\
\text { sanas. }\end{array}$ & $\begin{array}{l}p>0.05 \text { en la FCF en } \\
\text { reposo; } p<0.05 \text { en tiempo } \\
\text { de recuperación en FCF. }\end{array}$ & Moderada & $\begin{array}{l}\text { El EF mejora el sistema } \\
\text { cardiovascular y SNA en los } \\
\text { fetos de embarazadas que } \\
\text { realizan EF }\end{array}$ & Reoyo 2015. \\
\hline $\begin{array}{l}\text { Analizar la seguridad del EF aeróbico } \\
\text { para el sistema cardiovascular de las } \\
\text { gestantes. }\end{array}$ & $\begin{array}{l}45 \text { mujeres entre } \\
20 \text { y } 34 \text { semanas } \\
\text { de gestación }\end{array}$ & $\begin{array}{l}\text { Mejora en IMC y PA en } \\
\text { la semana } 20 \text { y aumento } \\
\text { de VS en la semana } 34 .\end{array}$ & Moderada & $\begin{array}{l}\text { El EF aeróbico controlado no } \\
\text { representa ningún riesgo en } \\
\text { embarazo. }\end{array}$ & $\begin{array}{l}\text { Perales et al., } \\
2015 .\end{array}$ \\
\hline
\end{tabular}

Nota: $\mathrm{AF}=$ actividad física; $\mathrm{EF}=$ ejercicio físico; $\mathrm{FCF}=$ frecuencia cardiaca fetal; $\mathrm{SNA}=$ sistema nervioso autónomo; $\mathrm{CF}=$ condición física; $\mathrm{VS}=$ volumen sistólico; $\mathrm{VO} \mathrm{O}_{2}=$ consumo de oxigeno; $\mathrm{PC}=$ peso corporal. 


\section{Académica sin Frontera}

ISSN: 2007-8870

\section{Revista de Investigación}

\section{http://revistainvestigacionacademicasinfrontera.com}

Principales efectos cardiovasculares que ocasiona el ejercicio físico en mujeres gestantes (continuación Tabla 1).

\begin{tabular}{|c|c|c|c|c|c|}
\hline Objetivo & Muestra & $\begin{array}{c}\text { Principales } \\
\text { resultados }\end{array}$ & $\begin{array}{l}\text { Intensidad de } \\
\text { entrenamiento }\end{array}$ & $\begin{array}{c}\text { Principales } \\
\text { Conclusiones }\end{array}$ & Autor/es y año \\
\hline $\begin{array}{l}\text { Conocer la influencia del EF materno } \\
\text { en bicicleta estática durante el } \\
\text { embarazo en el comportamiento } \\
\text { cardiocirculatorio materno-fetal, y la } \\
\text { respuesta del feto en FC. }\end{array}$ & $\begin{array}{l}20 \text { gestantes en el } \\
\text { último trimestre }\end{array}$ & $\begin{array}{l}\text { Aumenta la FCF de } 11- \\
\text { 36lat } / \mathrm{min}, p>0.05 \text { en la } \\
\text { arteria cerebral media } \\
\text { fetal antes ni después } \\
\text { del EF. }\end{array}$ & Moderado & $\begin{array}{l}\text { El EF aumenta la FCF sin } \\
\text { ningún efecto adverso, la arteria } \\
\text { cerebral fetal establece cambios } \\
\text { mínimos, estos cambios se } \\
\text { muestran dentro de los } \\
\text { estándares normales. }\end{array}$ & $\begin{array}{l}\text { Barakat Carballo, } \\
\text { López Mas, y } \\
\text { Montejo Rodríguez, } \\
2010 .\end{array}$ \\
\hline $\begin{array}{l}\text { Mostrar el comportamiento de } \\
\text { la CC y el perfil de lípidos en } \\
\text { mujeres en el } 3^{\text {er }} \text { trimestre de } \\
\text { gestación que realizan EF con baja } \\
\text { intensidad. }\end{array}$ & $\begin{array}{l}36 \text { gestantes } \\
\text { aparentemente sanas, } \\
\text { en periodo gestacional } \\
\text { de } 33 \text { a } 42 \text { semanas. }\end{array}$ & $\begin{array}{l}\text { Existe una relación } \\
\text { directa de la CC y el } \\
\text { perfil de lípidos con el } \\
\text { IMC pre- gestacional y } \\
\text { la poca actividad de } \\
\text { EF. }\end{array}$ & Baja & $\begin{array}{l}\text { La inactividad o la poca AF en el } \\
\text { embarazo podría ser un } \\
\text { determinante para disminuir la } \\
\text { incidencia de cesáreas en las } \\
\text { embarazadas. }\end{array}$ & $\begin{array}{l}\text { González } \\
\text { Vargas, et al., } 2014 .\end{array}$ \\
\hline
\end{tabular}

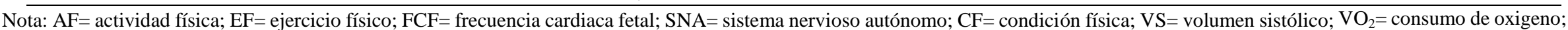
$\mathrm{PC}=$ peso corporal. 
Año 11.

Núm. 27

\section{Académica sin Frontera}

ISSN: 2007-8870

http://revistainvestigacionacademicasinfrontera.com

Revista de Investigación

Tabla 2. Principales efectos antropométricos que ocasiona el ejercicio físico en mujeres gestantes.

\begin{tabular}{|c|c|c|c|c|c|}
\hline Objetivo & Muestra & $\begin{array}{c}\text { Efecto del } \\
\text { ejercicio físico }\end{array}$ & $\begin{array}{l}\text { Intensidad de } \\
\text { entrenamiento }\end{array}$ & $\begin{array}{c}\text { Principales } \\
\text { conclusiones }\end{array}$ & Autor/es y año \\
\hline $\begin{array}{l}\text { Identificar los factores antropométricos } \\
\text { que influyen en el PC al nacer y su } \\
\text { relación con el EF. }\end{array}$ & $\begin{array}{l}140 \text { gestantes } \\
\text { obesas. }\end{array}$ & $\begin{array}{l}\text { El PC en neonatos de } \\
\text { madres obesas fue } \\
\text { mayor que en neonatos } \\
\text { de madres que } \\
\text { realizaron EF y } \\
\text { conservaron su IMC } \\
\text { normal. }\end{array}$ & Moderada & $\begin{array}{l}\text { El IMC pregestacional materno es } \\
\text { el predictor que mejor se asocia } \\
\text { con el PC del neonato. }\end{array}$ & $\begin{array}{l}\text { Vila Candel et al., } \\
2016 .\end{array}$ \\
\hline $\begin{array}{l}\text { Describir las experiencias de las } \\
\text { mujeres sobre la información } \\
\text { dietética, el EF y el cambio de } \\
\text { hábitos alimenticios durante el } \\
\text { embarazo. }\end{array}$ & $\begin{array}{l}23 \text { mujeres en la } \\
\text { mitad del periodo de } \\
\text { gestación. }\end{array}$ & $\begin{array}{l}\text { Las gestantes no } \\
\text { contaban con la } \\
\text { información } \\
\text { nutricional necesaria, el } \\
\text { EF era bajo y solamente } \\
\text { se realizaba cuando } \\
\text { alguna molestia física se } \\
\text { hacía presente. }\end{array}$ & Baja & $\begin{array}{l}\text { Es poca la desinformación sobre } \\
\text { la alimentación y el EF, no causa } \\
\text { impacto entre las gestantes. Se } \\
\text { continúa con hábitos inadecuados } \\
\text { durante el embarazo. }\end{array}$ & $\begin{array}{l}\text { Wennberg et al., } \\
2013 \text {. }\end{array}$ \\
\hline $\begin{array}{l}\text { Conocer el efecto del tiempo de } \\
\text { práctica de EF y las horas semanales } \\
\text { de práctica sobre algunos aspectos } \\
\text { cognitivos, emocionales y } \\
\text { antropométricos. }\end{array}$ & $\begin{array}{l}250 \text { mujeres, edad } \\
49 \text { años. }\end{array}$ & $\begin{array}{l}\text { El EF semanal } \\
\text { predice parte de la } \\
\text { varianza del PC, } \\
\text { estabilidad } \\
\text { emocional, } \\
\text { autoconcepto y } \\
\text { autoestima. }\end{array}$ & Moderada & $\begin{array}{l}\text { Entre mayor sea la de práctica } \\
\text { de EF, mejores son los } \\
\text { resultados de control de PC y } \\
\text { estabilidad emocional. }\end{array}$ & Zarceño, 2014. \\
\hline
\end{tabular}

Nota: $\mathrm{PC}=$ peso corporal $; \mathrm{IMC}=$ índice de masa corporal; $\mathrm{EF}=$ ejercicio físico. 
Año 11.

Núm. 27

\section{Académica sin Frontera \\ ISSN: 2007-8870}

http://revistainvestigacionacademicasinfrontera.com

Revista de Investigación

Principales efectos antropométricos que ocasiona el ejercicio físico en mujeres gestantes (continuación Tabla 2).

\begin{tabular}{|c|c|c|c|c|c|}
\hline Objetivo & Muestra & $\begin{array}{c}\text { Efecto del } \\
\text { ejercicio físico }\end{array}$ & $\begin{array}{l}\text { Intensidad de } \\
\text { entrenamiento }\end{array}$ & $\begin{array}{c}\text { Principales } \\
\text { conclusiones }\end{array}$ & Autor/es y año \\
\hline $\begin{array}{l}\text { Revisar las diferencias étnicas en: } \\
\text { adiposidad, hiperglucemia y preclamsia } \\
\text { durante el embarazo en mujeres que } \\
\text { realizan poca AF. }\end{array}$ & $\begin{array}{l}100 \text { embarazadas } \\
\text { sanas con edades de } \\
25 \text { a } 30 \text { años }\end{array}$ & $\begin{array}{l}\text { Se encuentra mayor } \\
\text { IMC en mujeres de } \\
\text { ascendencia africana } \\
\text { que son inactivas, } \\
\text { tienen más riesgo de } \\
\text { preeclamsia y obesidad } \\
\text { tardía en los neonatos. }\end{array}$ & Baja & $\begin{array}{l}\text { Existe mayor riesgo de } \\
\text { padecer obesidad y } \\
\text { preclamsia en africanas, y para sus } \\
\text { descendientes mayor riesgo de } \\
\text { obesidad tardía. }\end{array}$ & $\begin{array}{l}\text { Jenum et } \\
\text { al., } 2013 \text {. }\end{array}$ \\
\hline $\begin{array}{l}\text { Determinar si la implementación de una } \\
\text { dieta personalizada durante tres meses } \\
\text { mejora el estado de nutrición en gestantes } \\
\text { con sobrepeso y obesidad. }\end{array}$ & $\begin{array}{l}53 \text { gestantes } \\
\text { diagnosticadas con } \\
\text { sobrepeso y } \\
\text { obesidad. }\end{array}$ & $\begin{array}{l}\text { Se intervino a las } \\
\text { gestantes con dieta y EF } \\
\text { de relajación. Se } \\
\text { obtuvieron mejoras en } \\
\text { PC, PCI e indicadores } \\
\text { bioquímicos. }\end{array}$ & Baja & $\begin{array}{l}\text { La implementación del } \\
\text { programa de EF y dieta } \\
\text { personalizada, mejorando las } \\
\text { variables atendidas. }\end{array}$ & $\begin{array}{l}\text { Sandoval, Nieves, } \\
\text { Luna, } 2016 .\end{array}$ \\
\hline $\begin{array}{l}\text { Determinar relaciones del estado } \\
\text { nutricional pre-gestacional y la ganancia } \\
\text { de PC gestacional con la retención de PC } \\
\text { post-parto como factor de riesgo } \\
\text { cardiovascular. }\end{array}$ & 29 mujeres sanas. & $\begin{array}{l}\text { El IMC pre- } \\
\text { gestacional } \\
\text { incrementa los } \\
\text { valores normales. } \\
\text { El PC post-parto y el } \\
35 \% \text { de las gestantes } \\
\text { resultó ser pre- } \\
\text { hipertensas. }\end{array}$ & Baja & $\begin{array}{l}\text { La ganancia de PC excesiva en } \\
\text { el embarazo se relacionó } \\
\text { directamente con la retención } \\
\text { de PC postparto. El ICC es } \\
\text { importante para detectar riesgo } \\
\text { cardiovascular es en } \\
\text { gestantes. }\end{array}$ & $\begin{array}{l}\text { Orozco Muñoz et } \\
\text { al., } 2016 .\end{array}$ \\
\hline $\begin{array}{l}\text { Determinar el efecto, duración y } \\
\text { calidad de sueño con ganancia de PC y } \\
\text { complicaciones durante el embarazo. }\end{array}$ & $\begin{array}{l}203 \text { embarazadas en } \\
\text { el } 3^{\mathrm{er}} \text { trimestre. }\end{array}$ & $\begin{array}{l}\text { Gestantes inactivas y } \\
\text { con problemas de } \\
\text { sueño indicaron } \\
\text { ganancia de PC } \\
\text { durante el embarazo. }\end{array}$ & Baja & $\begin{array}{l}\text { La falta de sueño y la poca AF } \\
\text { resultaron ser un determinante } \\
\text { para ganar PC materno. }\end{array}$ & $\begin{array}{l}\text { García Chán, } \\
2014 .\end{array}$ \\
\hline
\end{tabular}

Nota: $\mathrm{PC}=$ peso corporal; $\mathrm{IMC}=$ índice de masa corporal; $\mathrm{EF}=$ ejercicio físico. 


\section{Académica sin Frontera}

Revista de Investigación

\section{ISSN: 2007-8870 \\ http://revistainvestigacionacademicasinfrontera.com}

Principales efectos antropométricos que ocasiona el ejercicio físico en mujeres gestantes (continuación Tabla 2).

\begin{tabular}{|c|c|c|c|c|c|}
\hline Objetivo & Muestra & $\begin{array}{c}\text { Efecto del } \\
\text { ejercicio físico }\end{array}$ & $\begin{array}{l}\text { Intensidad de } \\
\text { entrenamiento }\end{array}$ & $\begin{array}{c}\text { Principales } \\
\text { conclusiones }\end{array}$ & Autor/es y año \\
\hline $\begin{array}{l}\text { Evaluar la eficacia de un } \\
\text { programa de EF regular en la } \\
\text { prevención de depresión en } \\
\text { gestantes con sobrepeso y } \\
\text { obesidad. }\end{array}$ & $\begin{array}{l}106 \text { gestantes con } \\
\text { sobrepeso y } \\
\text { obesidad, edad de } \\
32 \text { a } 39 \text { años. }\end{array}$ & $\begin{array}{l}\text { El programa de EF } \\
\text { benefició a las } \\
\text { embarazadas } \\
\text { manteniendo el PC } \\
\text { adecuado en } 3 \text { er } \\
\text { trimestre reduciendo la } \\
\text { depresión. }\end{array}$ & Moderada & $\begin{array}{l}\text { Un programa de EF durante el } \\
\text { embarazo puede evitar la } \\
\text { ganancia de excesivo PC y } \\
\text { minimiza la depresión en el } 3^{\mathrm{er}} \\
\text { trimestre. }\end{array}$ & $\begin{array}{l}\text { Perales et al., } \\
2015 .\end{array}$ \\
\hline $\begin{array}{l}\text { Analizar los principales estudios sobre } \\
\text { la influencia de la AF sobre los } \\
\text { parámetros materno-fetales en el } \\
\text { medio acuático. }\end{array}$ & $\begin{array}{l}\text { Se identificaron } \\
45 \text { artículos a } \\
\text { través de } \\
\text { búsqueda en las } \\
\text { bases de datos } \\
\text { PUBMED, } \\
\text { Scopus y Google } \\
\text { Scholar. }\end{array}$ & $\begin{array}{l}\text { El EF acuático en el } \\
\text { embarazo tiene efectos } \\
\text { positivos respiratorios, } \\
\text { cardiovasculares y } \\
\text { musculares en la } \\
\text { gestante y el feto. }\end{array}$ & Moderada & $\begin{array}{l}\text { La información disponible } \\
\text { sobre los efectos del EF en el } \\
\text { embarazo pueden ser el primer } \\
\text { paso para continuar con la } \\
\text { investigación de su impacto en } \\
\text { el medio acuático }\end{array}$ & $\begin{array}{l}\text { Aguilar Cordero et } \\
\text { al., } 2014 \text {. }\end{array}$ \\
\hline $\begin{array}{l}\text { Cuantificar la relación existente entre } \\
\text { las medidas antropométricas de los } \\
\text { neonatos varones con la antropometría } \\
\text { materna. }\end{array}$ & $\begin{array}{l}259 \text { Neonatos, } \\
\text { madres con menos } \\
\text { de } 21 \text { años. }\end{array}$ & $\begin{array}{l}\text { La edad gestacional se } \\
\text { relacionó directamente } \\
\text { con el PC en los niños y } \\
p<0.05 \text { en PC neonatal } \\
\text { con IMC al final de la } \\
\text { gestación. }\end{array}$ & Baja & $\begin{array}{l}\text { La estatura, PC y perímetro } \\
\text { cefálico se relacionaron } \\
\text { directamente con la edad } \\
\text { gestacional e IMC. }\end{array}$ & $\begin{array}{l}\text { Granda y Granda } \\
2016 .\end{array}$ \\
\hline
\end{tabular}

Nota: $\mathrm{PC}=$ peso corporal $; \mathrm{IMC}=$ índice de masa corporal; $\mathrm{EF}=$ ejercicio físico. 
Año 11. Frontera

Núm. 27
Revista de Investigación Académica sin

ISSN: 2007-8870

\section{http://revistainvestigacionacademicasinfrontera.com}

\section{Discusiones}

En el presente estudio se analizaron los efectos y las consecuencias que se tiene el EF en parámetros antropométricos y cardiovasculares durante el periodo de gestación. Existe información que muestran los beneficios que genera realizar EF a las gestantes, incluyendo la prevención de diversas alteraciones metabólicas que directamente llegan a incidir en aumento de la grasa corporal, dolores articulares, fatiga muscular y dificultad para respirar (Broberg et al., 2016; Ewton y May, 2017; Feldens et al., 2010; Rodríguez-Blanque et al., 2017; Richardsen et al., 2016). Sanabria- Martínez et al. (2015); Oliveria et al. (2012); Church et al. (2010) demostraron que realizar EF durante el periodo de gestación 69\% la glucemia, disminuyendo la necesidad de insulina debido a que se aumenta la utilización de glucosa para cumplir con las demandas energéticas en los músculos activos. Está demostrado que la gonadotropina presente en la placenta puede generar bloqueo a la insulina generando diabetes gestacional (Sánchez, 2016).

Moyer et al. (2015) y Ong et al. (2016) demostraron que la práctica regular de EF contribuye en la mejora del estado de salud en mujeres embarazadas al fortalecer los músculos que están implicados al momento del parto. Esto puede ser consecuencia del constante trabajo físico que se somete la gestante e intensidad de entrenamiento aunado al consumo alimentario en los diversos trimestres de gestación. Rodríguez-Blanque et al. (2017) refieren que el EF realizado durante el periodo de gestación no presenta efectos negativos cuando se entrena a intensidades moderadas, bajo prescripción médica y deportiva. Sin embargo, autores como Nascimento, Surita, y Cecatti (2012) y Melzer et al. (2010) refieren que el EF durante este periodo se debe evitar en enfermedades coronarias, pulmonares e hipertensión gestacional o cuando presentan cérvix incompetente, gestación múltiple con riesgo de parto prematuro, sangrado persistente en el segundo o tercer trimestre. 
Año 11. Frontera

Núm. 27
Revista de Investigación Académica sin

ISSN: 2007-8870

\section{http://revistainvestigacionacademicasinfrontera.com}

Rodríguez-Blanque et al. (2017) realizaron un estudio en 140 gestantes sanas, cuyas edades oscilaban entre 21 y 43 años con la finalidad de analizar la influencia de un programa de AF moderada realizada en el medio acuático y su relación con la ventilación pulmonar, concluyendo que el EF en este medio no compromete la oxigenación pulmonar materna ni fetal. Esto puede ser debido al control de la intensidad de entrenamiento que se lleva a cabo, tal como lo mencionan Oliveria et al., (2012) y Aguilar (2011) que el EF realizado a intensidades por debajo del 70\% $\mathrm{VO}_{2}$ máx genera que la gestante mejore la capacidad cardiovascular, incidiendo en que el feto adquiera mayor oxigenación. Vega et al. (2017) evaluaron en gestantes saludables el efecto del EF aeróbico sobre el $\mathrm{VO}_{2}$ en 64 gestantes saludables con rango de edad de 20 y 28 años concluyendo que el EF durante el embarazo funciona como una alternativa para prevenir patologías relacionadas con el sedentarismo. Autores como Miranda y Navío (2013) y Vega et al. (2017) han fundamentado que el tiempo recomendado para la práctica de EF se encuentra entre 30 a 40 minutos diarios. Las recomendaciones son debidas a los efectos a nivel celular que se generan bajo estas directrices, principalmente adquirir mejoras en la capacidad metabólica y cardiopulmonar, así como reducir el riesgo de padecer diabetes gestacional (Sui y Dodd, 2013). También se compromete positivamente al sistema músculo-esquelético generando que los músculos del piso pélvico se fortalezcan con el trabajo físico continuo evitando la incontinencia urinaria, la incontinencia anal y el prolapso de órganos pélvicos (Martínez, Ribera, Hipólito, y Allepuz, 2013). Otros autores Domingues, Matijasevich, y Barros (2009) y Korsten-Reck (2010) atribuyen que el EF genera mayor flexibilidad en ligamentos de la pelvis ocasionando un aumento en el diámetro del cuello del útero lo cual facilita el parto natural.

Stannard y Wing (2016) mencionan que la actividad regular de EF durante el embarazo facilita la aptitud física en las primeras etapas de vida. Dimmock et al. (2015) atribuyen efectos negativos en la salud de la gestante y posiblemente del feto cuando se mantienen 
Año 11. Frontera

Núm. 27
Revista de Investigación Académica sin

ISSN: 2007-8870

\section{http://revistainvestigacionacademicasinfrontera.com}

hábitos pocos saludables durante la etapa de gestación. Posiblemente las problemáticas sean ocasionadas por los cambios que se van ocasionando al paso de las generaciones en los genes (Sánchez, Yoldi, Zabalza, y Hernández, 2010). Asimismo, Constantinou, Oddie, y Watson (2015) explican que existe que refiere a la importancia que representa el EF durante el embarazo es saludable pudiendo ser una herramienta eficaz para controlar el PC excesivo y sus complicaciones durante este periodo. También Blaize, Newcomer, y Pearson (2015) investigaron el impacto que tiene el EF en mujeres con riesgo de una enfermedad crónica en periodo gestacional demostrando mejoras metabólicas, salud cardiovascular.

\section{Conclusiones}

El EF practicado bajo prescripción médica y deportiva genera múltiples beneficios en la salud de la gestante y feto.

El predominio de las intensidades de entrenamiento no debe sobrepasar del $75 \%$ del $\mathrm{VO}_{2} \mathrm{máx}_{\text {, }}$ aunque según la aptitud física y condición de salud la gestante puede realizar sesiones por encima de este umbral.

Las gestantes que realizan EF controlado y adecuado presentan menor aumento de peso graso que las gestantes que no realizan estos programas.

El EF debe evitarse en condiciones de enfermedad crónicas o complicaciones como sangrado vaginal, disnea, dolor en el pecho, dolor de cabeza, mareo o vértigo. 
Año 11.

Frontera

Núm. 27
Revista de Investigación

Académica sin

ISSN: 2007-8870

\section{http://revistainvestigacionacademicasinfrontera.com}

\section{Agradecimientos}

Los autores agradecen a la Universidad Estatal de Sonora por las facilidades brindadas para la realización del artículo.

\section{Referencias bibliográficas}

Aguilar Cordero, M. J., Sánchez López, A. M., Rodríguez Blanque, R., Noack Segovia, J. P., Cano, P., López-Contreras, G., y Mur Villar, N. (2014). Actividad física en embarazadas y su influencia en parámetros materno-fetales: revisión sistemática. Nutrición Hospitalaria, 30(4), 719-726.

American College of Obstetricians and Gynecologists. (2013). Hypertension in pregnancy. Report of the American College of Obstetricians and Gynecologists' task force on hypertension in pregnancy. Obstetrics and gynecology, 122(5), 1122.

Barakat Carballo, R., López Mas, C., y Montejo Rodríguez, R. (2010) Influencia del ejercicio físico en el tercer trimestre del embarazo sobre el comportamiento cardiocirculatorio de la unidad materno-fetal. Revista Andaluza de Medicina del Deporte, 2010, vol. 3 , no 2 .

Bardosono, S., Morin, C., Guelinckx, I., y Pohan, R. (2017). Pregnant and Breastfeeding Women: Drinking for Two?. Annals of Nutrition and Metabolism, 70(Suppl. 1), 1317.

Bianchi, C. M., Huneau, J. F., Le Goff, G., Verger, E. O., Mariotti, F., y Gurviez, P. (2016). Concerns, attitudes, beliefs and information seeking practices with respect to nutrition-related issues: a qualitative study in French pregnant women. BMC pregnancy and childbirth, 16(1), 306.

Blaize, A. N., Pearson, K. J., y Newcomer, S. (2015). Impact of maternal exercise during pregnancy on offspring chronic disease susceptibility. Exercise and sport sciences reviews, 43(4), 198.

Bliss, C. I. (2014). The statistics of bioassay: with special reference to the vitamins. Elsevier.

Bookari, K., Yeatman, H., y Williamson, M. (2017). Falling short of dietary guidelines-What do Australian pregnant women really know? A cross sectional study. Women and Birth, 30(1), 9-17.

Borelli, M. F., Mayorga, M., Vega, S. M. D. L., Contreras, N. B., Tolaba, A. M., y Passamai, M. I. (2016). Estado nutricional y percepción de la imagen corporal de embarazadas 
Año 11. Frontera

Núm. 27
Revista de Investigación Académica sin

ISSN: 2007-8870

\section{http://revistainvestigacionacademicasinfrontera.com}

asistidas en centros de salud de Salta Capital, Argentina. Revista Española de Nutrición Humana y Dietética, 20(3), 174-179.

Bove, I., Mardones Santander, F., Klaps, L., y Domínguez de Landa, A. (2014). Asociaciones entre el crecimiento prenatal y la antropometría materna en el Uruguay. Nutrición Hospitalaria, 30(3), 643-649.

Broberg, L., Ersbøll, A. S., Backhausen, M. G., Damm, P., Tabor, A., y Hegaard, H. K. (2015). Compliance with national recommendations for exercise during early pregnancy in a Danish cohort. BMC pregnancy and childbirth, 15(1), 317.

Brown, A., y Avery, A. (2012). Healthy weight management during pregnancy: what advice and information is being provided. Journal of Human Nutrition and Dietetics, 25(4), 378-387.

Cerrato-López, C., Arroyo-Rodríguez, P., Cabrera-Martos, I., Torres-Sánchez, I., ChecaMoreno, V., y Valenza, M. C. (2017). Modificaciones musculoesqueléticas en mujeres embarazadas con dolor lumbopélvico. Fisioterapia, 39(1), 18-24.

Chen, X., Scholl, T. O., Stein, T. P., Steer, R. A., y Williams, K. P. (2017). Maternal circulating lipid profile during early pregnancy: Racial/ethnic differences and association with spontaneous preterm delivery. Nutrients, 9(1), 19.

Chia, L. C., Guelfi, K. J., y Licari, M. K. (2010). A comparison of the oxygen cost of locomotion in children with and without developmental coordination disorder. Developmental Medicine \& Child Neurology, 52(3), 251-255

Church, T. S., Blair, S. N., Cocreham, S., Johannsen, N., Johnson, W., Kramer, K., ... y Sparks, L. (2010). Effects of aerobic and resistance training on hemoglobin A1c levels in patients with type 2 diabetes: a randomized controlled trial. Jama, 304(20), 22532262

Cintas, P., Mantegna, S., Gaudino, E. C., y Cravotto, G. (2010). A new pilot flow reactor for high-intensity ultrasound irradiation. Application to the synthesis of biodiesel. Ultrasonics Sonochemistry, 17(6), 985-989.

Cordero, M. J. A., López, A. M. S., Baños, N. M., Villar, N. M., Ruiz, M. E., y Rodríguez, E. H. (2015). Lactancia materna como prevención del sobrepeso y la obesidad en el niño y el adolescente; revisión sistemática. Nutr Hosp, 31(2), 606-620.

Cruz Almaguer, C. D. L. C., Cruz Sánchez, L., López Menes, M., y González, J. D. (2012). Nutrición y embarazo: algunos aspectos generales para su manejo en la atención primaria de salud. Revista Habanera de Ciencias Médicas, 11(1), 168-175. 


\section{http://revistainvestigacionacademicasinfrontera.com}

Davies, G. A., Wolfe, L. A., Mottola, M. F., y MacKinnon, C. (2003). Joint SOGC/CSEP clinical practice guideline: exercise in pregnancy and the postpartum period. Canadian Journal of Applied Physiology, 28(3), 329-341.

Domingues, M. R., Matijasevich, A., y Barros, A. J. (2009). Physical activity and preterm birth. Sports medicine, 39(11), 961-975.

Drehmer, M., Duncan, B. B., Kac, G., y Schmidt, M. I. (2013). Association of second and third trimester weight gain in pregnancy with maternal and fetal outcomes. PloSone, $8(1)$, e 54704.

Drenthen, W., Pieper, P. G., Roos-Hesselink, J. W., van Lottum, W. A., Voors, A. A., Mulder, B. J., ... y Ebels, T. (2007). Outcome of pregnancy in women with congenital heart disease: a literature review. Journal of the American College of Cardiology, 49(24), 2303-2311.

Duvekot, J. J., Cheriex, E. C., Pieters, F. A., Menheere, P. P., y Peeters, L. L. (1993). Early pregnancy changes in hemodynamics and volume homeostasis are consecutive adjustments triggered by a primary fall in systemic vascular tone. American Journal of Obstetrics \& Gynecology, 169(6), 1382-1392.

Ewton, E. R., y May, L. (2017). Adaptation of maternal-fetal physiology to exercise in pregnancy: the basis of guidelines for physical activity in pregnancy. Clinical Medicine Insights: Women's Health, 10, 1179562X17693224.

Feldens, C. A., Giugliani, E. R. J., Duncan, B. B., Drachler, M. D. L., y Vítolo, M. R. (2010). Long- term effectiveness of a nutritional program in reducing early childhood caries: a randomized trial. Community dentistry and oral epidemiology, 38(4), 324-332.

Ferolla, F. M., Hijano, D. R., Acosta, P. L., Rodríguez, A., Dueñas, K., Sancilio, A., ... y Castro, L. (2013). Macronutrients during pregnancy and life-threatening respiratory syncytial virus infections in children. American journal of respiratory and critical care medicine, 187(9), 983-990.

Ferraro, Z. M., Gaudet, L., y Adamo, K. B. (2012). The potential impact of physical activity during pregnancy on maternal and neonatal outcomes. Obstetrical \& gynecological survey, 67(2), 99-110.

Firman, G. (2000). Fisiología del ejercicio físico. Corrientes, Argentina: Facultad de Medicina de la UNNE.

Flynn, A. C., Seed, P. T., Patel, N., Barr, S., Bell, R., Briley, A. L., ... y Sanders, T. A. (2016). Dietary patterns in obese pregnant women; influence of a behavioral intervention of 
Año 11. Frontera

Núm. 27
Revista de Investigación Académica sin

ISSN: 2007-8870

\section{http://revistainvestigacionacademicasinfrontera.com}

diet and physical activity in the UPBEAT randomized controlled trial. International Journal of Behavioral Nutrition and Physical Activity, 13(1), 124.

Gaibor, G., y Elizabeth, F. (2015). Conocimientos actitudes y prácticas que influyen en la realización del ejercicio físico en mujeres durante el segundo y tercer trimestre de gestación que acude a control de embarazo en el Hospital Gineco Obstétrico Isidro Ayora de la Ciudad de Quito, 2012.

Gale, C. R., Javaid, M. K., Robinson, S. M., Law, C. M., Godfrey, K. M., \& Cooper, C. (2007). Maternal size in pregnancy and body composition in children. The Journal of Clinical Endocrinology \& Metabolism, 92(10), 3904-3911.

García Chán, D. D. C. (2014). Duración y calidad del sueño con ganancia de peso y complicaciones del embarazo (Doctoral dissertation, Universidad Autónoma de Nuevo León).

Gaston, A., y Prapavessis, H. (2013). Tired, moody and pregnant? Exercise may be the answer. Psychology \& health, 28(12), 1353-1369.

González Vargas, D. J., Vásquez Garibay, E. M., Romero Velarde, E., Rolón Díaz, J. C., Troyo Sanromán, R., y Hidalgo Ornelas, J. D. J. (2014). Composición corporal y perfil de lípidos en mujeres en el tercer trimestre del embarazo.

González-Collado, F., Ruiz-Giménez, A., y Salinas-Salinas, G. J. (2013). Indicaciones y contraindicaciones del ejercicio físico en la mujer embarazada. Clínica e Investigación en Ginecología y Obstetricia, 40(2), 72-76.

Granda, R. D., y Granda, L. D. (2016). Estudio Transversal: Relación de la Antropometría Neonatal con la Edad Gestacional y Antropometría Materna. Hospital Vicente Corral. Cuenca-Ecuador. 2013. Revista Médica HJCA, 8(2), 117-122.

Guelfi, K. J., Wang, C., Dimmock, J. A., Jackson, B., Newnham, J. P., y Yang, H. (2015). A comparison of beliefs about exercise during pregnancy between Chinese and Australian pregnant women. BMC pregnancy and childbirth, 15(1), 345.

Guxens, M., Aguilera, I., Ballester, F., Estarlich, M., Fernández-Somoano, A., Lertxundi, A., ... y Sunyer, J. (2012). Prenatal exposure to residential air pollution and infant mental development: modulation by antioxidants and detoxification factors. Environmental health perspectives, 120(1), 144.

Hernández Díaz, D., Sarasa Muñoz, N. L., y Cañizares Luna, O. (2016). El índice de masa corporal puede no ser suficiente en el seguimiento ponderal de las gestantes. Medicentro Electrónica, 20(3), 209-212. 


\section{http://revistainvestigacionacademicasinfrontera.com}

Hernández, R. I. R., Quechol, G. R., Morales, H. R., y Pérez, R. (2005). Alimentación y estado nutricio de mujeres embarazadas derechohabientes del Instituto Mexicano del Seguro Social en un área suburbana de la Ciudad de México. Ginecol Obstet Mex, 73, 3-10.

Hui, A., Back, L., Ludwig, S., Gardiner, P., Sevenhuysen, G., Dean, H., ... y Murray, R. (2012). Lifestyle intervention on diet and exercise reduced excessive gestational weight gain in pregnant women under a randomised controlled trial. BJOG: An International Journal of Obstetrics \& Gynaecology, 119(1), 70-77.

Karen Jenum, A., Sommer, C., Sletner, L., Mørkrid, K., Bærug, A., y Mosdøl, A. (2013). Adiposity and hyperglycaemia in pregnancy and related health outcomes in European ethnic minorities of Asian and African origin: a review. Food \& nutrition research, 57(1), 18889.

Kisner, C., y Colby, L. A. (2005). Ejercicio terapéutico. Fundamentos y técnicas (Vol. 88). Editorial Paidotribo.

Korsten-Reck, U. (2010). Physical activity in pregnancy and in breast-feeding period in obese mothers. Zeitschrift Fur Geburtshilfe Und Neonatologie, 214(3), 95-102.

Ley, S. H., Hanley, A. J., Retnakaran, R., Sermer, M., Zinman, B., y O’Connor, D. L. (2011). Effect of macronutrient intake during the second trimester on glucose metabolism later in pregnancy-. The American journal of clinical nutrition, 94(5), 1232-1240.

Maldonado-Durán, M., Sauceda-García, J. M., y Lartigue, T. (2008). Cambios fisiológicos y emocionales durante el embarazo normal y la conducta del feto. Perinatol Reprod Hum, 22(1), 5-14.

Martínez, C. S. (2016). Realización de ejercicio físico durante el embarazo: beneficios y recomendaciones. Revista Española de Educación Física y Deportes, (414), 53-68.

Martínez, F. A., Ribera, E. P., Hipólito, M. A., y Allepuz, A. (2013). Impacto en la función de los músculos del suelo pélvico de un programa de entrenamiento específico incluido en el control habitual del embarazo y el posparto: ensayo clínico controlado no aleatorizado. Matronas profesión, (2), 36-44.

Mata, F., Chulvi, I., Roig, J., Heredia, J. R., Isidro, F., Benítez Sillero, J. D., y Guillén del Castillo, M. (2010). Prescripción del ejercicio físico durante el embarazo. Revista andaluza de medicina del deporte, 3(2). 


\section{http://revistainvestigacionacademicasinfrontera.com}

Matheson, G. O., Klügl, M., Engebretsen, L., Bendiksen, F., Blair, S. N., Börjesson, M., ... y Khan, K. M. (2013). Prevention and management of non-communicable disease: the IOC consensus statement, Lausanne 2013. Br J Sports Med, 47(16), 1003-1011.

McGowan, C. A., y McAuliffe, F. M. (2013). Maternal dietary patterns and associated nutrient intakes during each trimester of pregnancy. Public health nutrition, 16(1), 97-107.

Mead, G. E., Morley, W., Campbell, P., Greig, C. A., McMurdo, M., y Lawlor, D. A. (2009). Exercise for depression. Cochrane Database Syst Rev, 3.

Melzer, K., Schutz, Y., Boulvain, M., y Kayser, B. (2010). Physical activity and pregnancy. Sports Medicine, 40(6), 493-507.

Mendoza, L., Pérez, B., y Sánchez Bernal, S. (2010). Estado nutricional de embarazadas en el último mes de gestación y su asociación con las medidas antropométricas de sus recién nacidos. Pediatría (Asunción), 37(2), 91-96.

Miranda, M. D., y Navío, C. (2013). Benefits of exercise for pregnant women. Journal of Sport and Health Research, 5(2), 229-232.

Moran, L. J., Sui, Z., Cramp, C. S., y Dodd, J. M. (2013). A decrease in diet quality occurs during pregnancy in overweight and obese women which is maintained post-partum. International journal of obesity, 37(5), 704.

Mourtakos, S. P., Tambalis, K. D., Panagiotakos, D. B., Antonogeorgos, G., Arnaoutis, G., Karteroliotis, K., y Sidossis, L. S. (2015). Maternal lifestyle characteristics during pregnancy, and the risk of obesity in the offspring: a study of 5,125 children. BMC pregnancy and childbirth, 15(1), 66.

Moyer, C., Livingston, J., Fang, X., y May, L. E. (2015). Influence of exercise mode on pregnancy outcomes: ENHANCED by Mom project. BMC pregnancy and childbirth, 15(1), 133.

Moyer, C., Reoyo, O. R., y May, L. (2016). The Influence of Prenatal Exercise on Offspring Health: A Review. Clinical medicine insights. Women's health, 9, 37.

Muñoz, C. O., Muñoz, N. L. S., Luna, O. C., Díaz, D. H., Pérez, Y. L., y Díaz, B. M. (2016). Retención de peso postparto y riesgo cardiovascular. CorSalud, 8(2), 94-101.

Nascimento, S. L., Surita, F. G., Godoy, A. C., Kasawara, K. T., y Morais, S. S. (2015). Physical activity patterns and factors related to exercise during pregnancy: a cross sectional study. PloS one, 1 Barakat, R., Perales, M., Bacchi, M., Coteron, J., y 


\section{http://revistainvestigacionacademicasinfrontera.com}

Refoyo, I. (2014). A program of exercise throughout pregnancy. Is it safe to mother and newborn?. American journal of health promotion, 29(1), 2-8.0(6), e0128953.

Nelson, D. B., Hanlon, A., Nachamkin, I., Haggerty, C., Mastrogiannis, D. S., Liu, C., y

Fredricks, D. N. (2014). Early Pregnancy Changes in Bacterial Vaginosis- Associated Bacteria and Preterm Delivery. Paediatric and perinatal epidemiology, 28(2), 88-96. Nemati, T., y Zarezadeh, N. (2017). The effect of exercise on childbirth in primiparous women: A clinical trial study. Nursing Practice Today, 3(2), 70-78.

NORMA Oficial Mexicana NOM-043-SSA2-2012, Servicios básicos de salud. Promoción y educación para la salud en materia alimentaria. Criterios para brindar orientación.

Oliveria Melo, A. S., Silva, J. L. P., Tavares, J. S., Barros, V.O., Leite, D. F., y Amorim, M. M. (2012). Effect of a physical exercise program during pregnancy on uteroplacental and fetal blood flow and fetal growth: a randomized controlled trial. Obstetrics \& Gynecology, 120(2, Part 1), 302-310.

Ong, M. J., Wallman, K. E., Fournier, P. A., Newnham, J. P., y Guelfi, K. J. (2016). Enhancing energy expenditure and enjoyment of exercise during pregnancy through the addition of brief higher intensity intervals to traditional continuous moderate intensity cycling. BMC pregnancy and childbirth, 16(1), 161.

Onoyama, S., Qiu, L., Low, H. P., Chang, C. I., Strohsnitter, W. C., Norwitz, E. R., ... y Lagiou, P. (2016). Prenatal maternal physical activity and stem cells in umbilical cord blood. Medicine and science in sports and exercise, 48(1), 82.

Oppizzi, Y., y Chernovetzky, G. (2015). Incidencia de cardiopatías congénitas en una maternidad pública en los inicios del Programa Nacional de Cardiopatías Congénitas. Revista argentina de cardiología, 83(1), 42-48.

Orozco, L. T., Vergara, J. P., Ruíz, O. C., y Ayasta, C. (2015). Factores de Riesgo Asociados a la Hipertensión Inducida por el embarazo. Revista Peruana de Ginecología y Obstetricia, 39(17), 87-91.

Pendón, M. J. A., Muñoz, P. B., y Alés, M. D. M. (2016). CA 2-326: Los beneficios de la actividad física acuática durante el embarazo. Enfermería docente, 1(106), 183.

Perales Santaella, M., Cordero Rodriguez, Y., Vargas, M., Lucía Mulas, A., y Barakat Carballo, R. O. (2015). Exercise and depression in overweight and obese pregnant women: a randomised controlled trial. Archivos de Medicina del Deporte, 167, 7077. 
Año 11. Frontera

Núm. 27
Revista de Investigación

Académica sin

ISSN: 2007-8870

\section{http://revistainvestigacionacademicasinfrontera.com}

Pérez Guillén, A., y Bernal Rivas, J. (2006). Predicción del estado nutricional mediante variables antropométricas y de seguridad alimentaria en el hogar de un grupo de embarazadas de Caracas, Venezuela. Nutrición Hospitalaria, 21(5), 611-616.

Pescatello, L. S., Arena, R., Riebe, D., y Thompson, P. D. (2013). SNEAK PEEK: Preview of ACSM's Guidelines for Exercise Testing and Prescription. ACSM's Health \& Fitness Journal, 17(2), 16-20.

Petrov Fieril, K., Glantz, A., y Fagevik Olsen, M. (2015). The efficacy of moderate- tovigorous resistance exercise during pregnancy: a randomized controlled trial. Acta obstetricia et gynecologica Scandinavica, 94(1), 35-42.

Pomata, J., García, H., Otheguy, L., Aspres, N., Fernández, T., Ballicora, A., y González, M. (1997). Adolescencia y embarazo. Rev. Hosp. Mat. Inf. Ramón Sardá, 16(2), 52-56.

Ramírez-Palacios, L. A., Catacora-Navarro, M. O., Morales-Lima, K. V., HernándezPacheco, J. A., y Estrada-Altamirano, A. (2017). Riesgo de complicaciones maternas y perinatales en mujeres con coartación aórtica. Estudio de casos y controles. Ginecología y obstetricia de México, 85(1), 1-6.

Ravasco, P., Anderson, H., y Mardones, F. (2010). Métodos de valoración del estado nutricional. Nutrición Hospitalaria, 25, 57-66.

Reoyo, O. R. (2015). Influencia de un programa de ejercicio físico desarrollado durante el embarazo en la respuesta cardiaca fetal. Influence of a physical activity program performed during pregnancy on fetal heart response (Doctoral dissertation, Universidad Politécnica de Madrid).

Richardsen, K. R., Falk, R. S., Jenum, A. K., Mørkrid, K., Martinsen, E. W., Ommundsen, Y., y Berntsen, S. (2016). Predicting who fails to meet the physical activity guideline in pregnancy: a prospective study of objectively recorded physical activity in a population-based multi-ethnic cohort. BMC pregnancy and childbirth, 16(1), 186.

Richmond, R. C., Timpson, N. J., Felix, J. F., Palmer, T., Gaillard, R., McMahon, G., ... y Lawlor, D. A. (2017). Using genetic variation to explore the causal effect of maternal pregnancy adiposity on future offspring adiposity: a Mendelian Randomisation Study. PLoS medicine, 14(1), e1002221.

Rodríguez-Blanque, R., Sánchez-García, J. C., Sánchez-López, A. M., Mur-Villar, N., Fernández-Castillo, R., y Cordero, M. J. A. (2017). Influencia del ejercicio físico durante el embarazo sobre el peso del recién nacido: un ensayo clínico aleatorizado. Nutrición Hospitalaria, 34(4), 834-840. 


\section{http://revistainvestigacionacademicasinfrontera.com}

Roldan, O., Perales, M., Mateos, S., y Barakat, R. (2015). Supervised physical activity during pregnancy improves fetal cardiac response el ejercicio físico supervisado durante el embarazo mejora la respuesta cardiaca fetal. International Journal of Medicine and Science of Physical Activity and Sport, 15(60), 757-772.

Sahariah, S. A., Potdar, R. D., Gandhi, M., Kehoe, S. H., Brown, N., Sane, H., ... y Cox, V. A. (2016). A Daily Snack Containing Leafy Green Vegetables, Fruit, and Milk before and during Pregnancy Prevents Gestational Diabetes in a Randomized, Controlled Trial in Mumbai, India-4. The Journal of nutrition, 146(7), 1453S-1460S.

Sanabria- Martínez, G., García- Hermoso, A., Poyatos- León, R., Álvarez- Bueno, C Sánchez- López, M., y Martínez- Vizcaíno, V. (2015). Effectiveness of physical activity interventions on preventing gestational diabetes mellitus and excessive maternal weight gain: a meta- analysis. BJOG: An International Journal gobstetrics \& Gynaecology, 122(9), 1167-1174.

Sánchez García, J. C., Rodríguez Blanque, R., Sánchez López, A. M., Baena García, L., Suárez Manzano, S., y Aguilar Cordero, M. J. (2016). Efectos de la actividad física durante el embarazo y en la recuperación posparto: protocolo de estudio. Nutrición Hospitalaria, 33(5).

Sánchez, A. I., Bermúdez, M. P., y Buela-Casal, G. (1999). Efectos del estado de gestación sobre el estado de ánimo. Revista electrónica de motivación y emoción, 2(2-3).

Sánchez, P. C., Yoldi, F. I. M., Zabalza, J. C., y Hernández, J. A. M. (2010). Epigenética nutricional: una pieza clave en el rompecabezas de la obesidad. Revista Española de Obesidad• Vol, 8(1), 10-20.

Sánchez-García, J. C., Rodríguez-Blanque, R., Mur Villar, N., Sánchez-López, A. M., Levet Hernández, M. C., y Aguilar-Cordero, M. J. (2016). Influencia del ejercicio físico sobre la calidad de vida durante el embarazo y el posparto: revisión sistemática. Nutrición Hospitalaria, 33, 1-9

Sandoval, K. V., Nieves, E. R., y Luna, M. Á. (2016). Efecto de una dieta personalizada en mujeres embarazadas con sobrepeso u obesidad. Revista chilena de nutrición, 43(3), 233-246.

Sanghavi, M., y Rutherford, J. D. (2014). Cardiovascular physiology of pregnancy. Circulation, 130(12), 1003-1008. 


\section{http://revistainvestigacionacademicasinfrontera.com}

Santos, I. A., Stein, R., Fuchs, S. C., Duncan, B. B., Ribeiro, J. P., Kroeff, L. R., ... y Schmidt, M. I. (2005). Aerobic exercise and submaximal functional capacity in overweight pregnant women: a randomized trial. Obstetrics \& Gynecology, 106(2), 243-249.

Serrano, E., Rus, G., y Garcia-Martinez, J. (2009). Nanotechnology for sustainable energy. Renewable and Sustainable Energy Reviews, 13(9), 2373-2384.

Sim, A. Y., Wallman, K. E., Fairchild, T. J., y Guelfi, K. J. (2015). Effects of high-intensity intermittent exercise training on appetite regulation. Medicine \& Science in Sports \& Exercise, 47(11), 2441-2449

Smith, K. M., Foster, R. C., y Campbell, C. G. (2011). Accuracy of physical activity assessment during pregnancy: an observational study. BMC pregnancy and childbirth, $11(1), 86$.

Suárez González, J. A., Gutiérrez Machado, M., Cabrera Delgado, M. R., Corrales Gutiérrez, A., y Salazar, M. E. (2011). Predictores de la preeclampsia/eclampsia en un grupo de gestantes de alto riesgo. Revista Cubana de Obstetricia y Ginecología, 37(2), 154161.

Tang, H., Hammack, C., Ogden, S. C., Wen, Z., Qian, X., Li, Y., ... y Christian, K. M. (2016). Zika virus infects human cortical neural progenitors and attenuates their growth. Cell stem cell, 18(5), 587-590.

Tijerina Sáenz, A., Ramírez López, E., Meneses Valderrama, V.M., y Martínez Garza, N. E. (2014). Ingesta energética y de macronutrientes en mujeres embarazadas en el noreste de México. Archivos Latinoamericanos de Nutrición, 64(3), 174-181.

Toirac Lamarque, A. S., Pascual López, V., Betancourt, D., Mastrapa Cantillo, K., y Torres González, Y. (2010). Hipertensión arterial durante el embarazo, el parto y el puerperio. Medisan, 14(5), 0-0.

Torres-Luque, G., López-Fernández, I., Santos-Lozano, A., Garatachea, N., y Álvarez Carnero, E. (2015). Actividad física y acelerometría; orientaciones metodológicas, recomendaciones y patrones. Nutrición Hospitalaria, 31(1).

Valdés, R., y Carvajal, J. A. (2016). Ensayo randomizado del uso de pesario cervical para prevenir parto prematuro en embarazos únicos (1). Revista chilena de obstetricia y ginecología, 81(3), 262-264.

Valdivia, S., Bruno, A., y Romero, M. (2017). Características antropométricas maternas y del lactante, correlacionado a la concentración proteica del calostro y leche madura: un estudio longitudinal. Revista chilena de obstetricia y ginecología, 82(2), 50-57. 


\section{http://revistainvestigacionacademicasinfrontera.com}

Valera, J. (2009). El embarazo en la adolescencia. Impresión digital o electrónica.

Vega, L. L., Castro, A. D., Ermeso, G. A., Ygualada, J. A. S., Ygualada, S. S., y Fernández, E. G. (2017). Cardiopatías asociadas a síndromes genéticos diagnosticados en pacientes pediátricos. Acta Médica del Centro, 11(4), 1-12.

Ventura, S. J., Curtin, S. C., Abma, J. C., y Henshaw, S. K. (2012). Estimated pregnancy rates and rates of pregnancy outcomes for the United States, 1990-2008. National vital statistics reports: From the centers for disease control and prevention, National Center for Health Statistics, National Vital Statistics System, 60(7), 1-21.

Vézina-Im, L. A., Godin, G., Couillard, C., Perron, J., Lemieux, S., y Robitaille, J. (2016). Validity and reliability of a brief self-reported questionnaire assessing fruit and vegetable consumption among pregnant women. BMC public health, 16(1), 982.

Vila Candel, R., Sanchis Valero, S., Mateu Ciscar, C., Bellvis Vázquez, E., Planells López, E., Martínez Ballester, A., ... y Espuig Sebastian, R. (2016). ¿Cuál es el mejor indicador antropométrico para el control del embarazo?. Nutrición Clínica y Dietética Hospitalaria, 36(3), 87-96.

Vohr, B. R., Davis, E. P., Wanke, C. A., y Krebs, N. F. (2017). Neurodevelopment: The Impact of Nutrition and Inflammation During Preconception and Pregnancy in LowResource Settings. Pediatrics, 139 (Supplement 1), 38-49.

Watson, E. D., Oddie, B., y Constantinou, D. (2015). Exercise during pregnancy: knowledge and beliefs of medical practitioners in South Africa: a survey study. BMC pregnancy and childbirth, 15(1), 245.

Weinberg, R. S. (2010). Fundamentos de psicología del deporte y del ejercicio físico. Ed. Médica Panamericana.

Wennberg, A. L., Lundqvist, A., Högberg, U., Sandström, H., y Hamberg, K. (2013). Women's experiences of dietary advice and dietary changes during pregnancy. Midwifery, 29(9), 1027-1034.

Wing, C. H., y Stannard, A. B. (2016). Pregnancy and Exercise Guidelines: Fifty Years Makes a Difference. ACSM's Health \& Fitness Journal, 20(2), 4-6.

Yazdkhasti, M., Pourreza, A., Pirak, A., y Abdi, F. (2015). Unintended pregnancy and its adverse social and economic consequences on health system: A narrative review article. Iranian journal of public health, 44(1), 12.

Zarceño-DOI, E. L., y Rosa, M. A. S. (2014). Ejercicio físico regular y variables cognitivas y emocionales en mujeres. Informació Psicològica, (108), 59-67. 